\title{
Heteroscedastic controlled calibration model applied to analytical chemistry
}

\author{
Betsabé G. Blas Achic and Mônica C. Sandoval \\ Departamento de Estatística, Universidade de São Paulo, São Paulo, Brasil
}

\begin{abstract}
In chemical analysis made by laboratories one has the problem of determining the concentration of a chemical element in a sample. In order to tackle this problem the guide EURACHEM/CITAC recommends the application of the linear calibration model, so implicitly assume that there is no measurement error in the independent variable $X$. In this work, it is proposed a new calibration model assuming that the independent variable is controlled. This assumption is appropriate in chemical analysis where the process tempting to attain the fixed known value $X$ generates an error and the resulting value is $x$, which is not an observable. However, observations on its surrogate $X$ are available. A simulation study is carried out in order to verify some properties of the estimators derived for the new model and it is also considered the usual calibration model to compare it with the new approach. Three applications are considered to verify the performance of the new approach.
\end{abstract}

Keywords: linear calibration model, controlled variable, measurement error model, uncertainty, chemical analysis. 


\section{Introduction}

The usual calibration model [1] is commonly used to estimate the concentration $X_{0}$ of a chemical species in a test sample. It typically assumes that the independent variable is fixed and it is not subject to error. However, in applications in analytical chemistry this variable is subject to error which arises from the preparation process of a standard solution. In many studies, such as [2], [3] and [4], it is attempted to consider the uncertainties due to the preparation process of the standard solutions by application of the error propagation law to the standard error of the estimator of $X_{0}$.

We have that the concentration of the standard solution is pre-fixed by the chemical analyst and a process is carried out attempting to attain it, this process generates errors. Hence, in this case it arises the so called controlled variable [5], where the controlled variable $X$ is defined by the pre-fixed concentration value of the standard solution which is expressed by the equation $X=x+\delta$, where $x$ is the unobserved variable and $\delta$ is the measurement error variable.

In [6] it was proposed the so called homoscedastic controlled calibration model. This model is formulated in the framework of the usual calibration model assuming that the independent variable is a controlled variable and the associated measurement errors have equal variances.

In [7] and [8], some methods to compute the uncertainties in certain values obtained through measurements are studied. In [8], the uncertainties of standard solutions are computed and it is observed that these uncertainties depend on the concentration values, so we can observe that the usual calibration model and the homoscedastic controlled calibration model seem not to be the more suitable ones. This problem motivates us to study a calibration model that considers the errors variability of the preparation of standard solutions. In this work we propose a calibration model that incorporates the errors variability arisen from the preparation process of the standard solution and we call it as the heteroscedastic controlled calibration model. This work is a continuation to our previous paper [6] in which it was undertaken the study of the so-called homoscedastic controlled calibration model which assumed equal variance errors.

The paper is organized as follows. In Section 2, we formulate the heteroscedastic controlled calibration model. In Section 3, a simulation study to test the new approach is presented. In Section 4, three applications are considered which show that the proposed model seems to be more adequate. Section 5 presents our concluding remarks. Finally, we present in Appendix A the usual calibration model, and in Appendix 3 some tables showing the results of the simulation study. 


\section{The proposed model}

Among the relevant problems in chemical analysis is the one related to the estimation of the concentration $X_{0}$ of a chemical compound in a given sample. In order to tackle this problem it is used a statistical calibration model, which is defined by a two-step process. This problem has been considered in [9] and [10].

The first stage of the calibration model is given by data points $(X, Y)$ which is determined in an experiment where the independent variable $X$ is the one that the experimenter selects. For instance, the concentrations of the standard solutions that a chemist prepares are independent variables since any concentration may be chosen. The dependent variable $Y$ is a measurable property of the independent variable. For example, the dependent variable may be the amount of intensity supplied by the plasma spectrometry method, since the intensity depends on the concentration.

In the second stage of the calibration model it is prepared a suitable sample related to the unknown concentration $X_{0}$ in order to obtain the measurements $Y_{0}$.

We have that the standard concentration $X$ is fixed by the analyst and the process of preparation attempting to get it produces an error $\delta$, and the unobserved quantity attained is $x$. Considering the usual calibration model defined by the equations A.1 and A.2 in the Appendix $\mathrm{A}$ and the equation $X=x+\delta$, we define the heteroscedastic controlled calibration model as

$$
\begin{aligned}
Y_{i} & =\alpha+\beta x_{i}+\epsilon_{i}, & i=1,2 \cdots, n, \\
X_{i} & =x_{i}+\delta_{i}, & i=1,2 \cdots, n, \\
Y_{0 i} & =\alpha+\beta X_{0}+\epsilon_{i}, & i=n+1, n+2, \cdots, n+k .
\end{aligned}
$$

It is considered the usual calibration model assumptions (see Appendix A) in addition to the following conditions

- $\delta_{1}, \delta_{2}, \cdots, \delta_{n}$ are independent and normally distributed with mean 0 .

- the variances $\sigma_{\delta_{i}}^{2},(i=1, \cdots, n)$ are supposed to be known.

- $\delta_{i}, i=1, \cdots, n$ and $\epsilon_{i}, i=1, \cdots, n+k$ are independent.

Observe that in the model described above we only consider the case when the variances $\sigma_{\delta_{i}}^{2}, i=1, \cdots, n$ are known. It is a generalization of the homoscedastic controlled calibration model discussed in [6], when it is considered $\sigma_{\delta_{i}}^{2}=\sigma_{\delta}^{2}$ for all $i$ and the known $\sigma_{\delta}^{2}$ case. This new model is also a generalization of the usual calibration model in which one takes $\delta_{i}=0, i=$ $1, \cdots, n$.

For the heteroscedastic controlled calibration model the logarithm of the likelihood function is given by

$$
l\left(\alpha, \beta, X_{0}, \sigma_{\epsilon}^{2}\right) \quad \propto-\frac{1}{2} \sum_{i=1}^{n} \log \left(\gamma_{i}\right)-\frac{k}{2} \log \left(\sigma_{\epsilon}^{2}\right)
$$




$$
-\frac{1}{2}\left[\sum_{i=1}^{n} \frac{\left(Y_{i}-\alpha-\beta X_{i}\right)^{2}}{\gamma_{i}}+\sum_{i=n+1}^{n+k} \frac{\left(Y_{0 i}-\alpha-\beta X_{0}\right)^{2}}{\sigma_{\epsilon}^{2}}\right]
$$

where $\gamma_{i}=\sigma_{\epsilon}^{2}+\beta^{2} \sigma_{\delta_{i}}^{2}, i=1, \cdots, n$. Solving $\partial l / \partial \alpha=0$ and $\partial l / \partial X_{0}=0$ one can get the maximum likelihood estimator of $\alpha$ and $X_{0}$ given, respectively, by

$$
\hat{\alpha}=\bar{Y}-\hat{\beta} \bar{X} \quad \text { and } \quad \hat{X}_{0}=\frac{\bar{Y}_{0}-\hat{\alpha}}{\hat{\beta}} .
$$

From (2.4) and (2.5), it follows that the logarithm of the likelihood function for $\left(\alpha, \beta, X_{0}, \sigma_{\epsilon}^{2}\right)$ can be writen as

$$
\begin{aligned}
l\left(\alpha, \beta, X_{0}, \sigma_{\epsilon}^{2}\right) & \propto-\frac{1}{2} \sum_{i=1}^{n} \log \left(\gamma_{i}\right)-\frac{k}{2} \log \left(\sigma_{\epsilon}^{2}\right) \\
& -\frac{1}{2}\left[\sum_{i=1}^{n} \frac{\left[\left(Y_{i}-\bar{Y}\right)-\beta\left(X_{i}-\bar{X}\right)\right]^{2}}{\gamma_{i}}+\frac{1}{\sigma_{\epsilon}^{2}} \sum_{i=n+1}^{n+k}\left(Y_{0 i}-\bar{Y}_{0}\right)^{2}\right] .
\end{aligned}
$$

Making $\partial l / \partial \beta=0, \partial l / \partial \sigma_{\epsilon}^{2}=0$ in the logarithm of the likelihood function (2.6), we have the following equations

$$
\begin{aligned}
\sum_{i=1}^{n} \frac{\beta \sigma_{\delta_{i}}^{2}\left[\gamma_{i}-\left(Y_{i}-\alpha-\beta X_{i}\right)^{2}\right]}{\gamma_{i}^{2}} & =\sum_{i=1}^{n} \frac{X_{i}\left(Y_{i}-\alpha-\beta X_{i}\right)}{\gamma_{i}} \\
\sum_{i=1}^{n} \frac{\gamma_{i}-\left(Y_{i}-\alpha-\beta X_{i}\right)^{2}}{\gamma_{i}^{2}} & =\sum_{i=n+1}^{n+k} \frac{\left(Y_{0 i}-\bar{Y}_{0}\right)^{2}}{\sigma_{\epsilon}^{4}}-\frac{k}{\sigma_{\epsilon}^{2}} .
\end{aligned}
$$

The estimates of $\beta$ and $\sigma_{\epsilon}^{2}$ can be obtained through some iterative method that solves the equations (2.7) and (2.8).

The Fisher expected information $I(\theta)=I\left(\alpha, \beta, X_{0}, \sigma_{\epsilon}^{2}\right)$ is given by

$$
I(\theta)=\left(\begin{array}{cccc}
\sum_{i=1}^{n} \frac{1}{\gamma_{i}}+\frac{k}{\sigma_{\epsilon}^{2}} & \sum_{i=1}^{n} \frac{X_{i}}{\gamma_{i}}+\frac{k X_{0}}{\sigma_{\epsilon}^{2}} & \frac{k \beta}{\sigma_{\epsilon}^{2}} & 0 \\
\sum_{i=1}^{n} \frac{X_{i}}{\gamma_{i}}+\frac{k X_{0}}{\sigma_{\epsilon}^{2}} & \sum_{i=1}^{n} \frac{X_{i}^{2}}{\gamma_{i}}+2 \beta^{2} \sum_{i=1}^{n} \frac{\sigma_{\delta_{i}}}{\gamma_{i}^{2}}+\frac{k X_{0}^{2}}{\sigma_{\epsilon}^{2}} & \frac{k \beta X_{0}}{\sigma_{\epsilon}^{2}} & \beta \sum_{i=1}^{n} \frac{\sigma_{i}^{2}}{\gamma_{i}^{2}} \\
\frac{k \beta}{\sigma_{\epsilon}^{2}} & \frac{k \beta X_{0}}{\sigma_{\epsilon}^{2}} & \frac{k \beta^{2}}{\sigma_{\epsilon}^{2}} & 0 \\
0 & \beta \sum_{i=1}^{n} \frac{\sigma_{\delta_{i}}^{2}}{\gamma_{i}^{2}} & 0 & \sum_{i=1}^{n} \frac{1}{2 \gamma_{i}^{2}}+\frac{k}{2 \sigma_{\epsilon}^{4}}
\end{array}\right)
$$

When $k=q n, q \in Q^{+}$and $n \rightarrow \infty$, the estimator $\hat{\theta}$ is approximately normally distributed with mean $\theta$ and variance $I(\theta)^{-1}$, thus the approximate variance to order $n^{-1}$ for $\hat{X}_{0}$ is given by

$$
V\left(\hat{X}_{0}\right)=\frac{\sigma_{\epsilon}^{2}}{\beta^{2}}\left[\frac{1}{n}+\frac{1}{k}-\frac{E_{1}}{n \sigma_{\epsilon}^{2} E_{2}}\right]
$$

where

$$
E_{1}=-n \sum_{i=1}^{n} \frac{X_{0}^{2} \sigma_{\epsilon}^{4}}{\gamma_{i}} \sum_{i=1}^{n} \frac{1}{\gamma_{i}^{2}}-n k \sum_{i=1}^{n} \frac{X_{0}^{2}}{\gamma_{i}}-n \sum_{i=1}^{n} \frac{X_{i}^{2} \sigma_{\epsilon}^{4}}{\gamma_{i}} \sum_{i=1}^{n} \frac{1}{\gamma_{i}^{2}}-n k \sum_{i=1}^{n} \frac{X_{i}^{2}}{\gamma_{i}}
$$




$$
\begin{aligned}
& -2 n \beta^{2} \sum_{i=1}^{n} \frac{\sigma_{\delta_{i}}^{4} \sigma_{\epsilon}^{4}}{\gamma_{i}^{2}} \sum_{i=1}^{n} \frac{1}{\gamma_{i}^{2}}-2 n k \beta^{2} \sum_{i=1}^{n} \frac{\sigma_{\delta_{i}}^{4}}{\gamma_{i}^{2}}+2 n \beta^{2} \sigma_{\epsilon}^{4}\left[\sum_{i=1}^{n} \frac{\sigma_{\delta_{i}}^{2}}{\gamma_{i}^{2}}\right]^{2} \\
& +2 n X_{0} \sigma_{\epsilon}^{4} \sum_{i=1}^{n} \frac{X_{i}}{\gamma_{i}} \sum_{i=1}^{n} \frac{1}{\gamma_{i}^{2}}+2 n k X_{0} \sum_{i=1}^{n} \frac{X_{i}}{\gamma_{i}}+\sigma_{\epsilon}^{6} \sum_{i=1}^{n} \frac{X_{i}^{2}}{\gamma_{i}} \sum_{i=1}^{n} \frac{1}{\gamma_{i}^{2}} \sum_{i=1}^{n} \frac{1}{\gamma_{i}} \\
& +k \sigma_{\epsilon}^{2} \sum_{i=1}^{n} \frac{X_{i}^{2}}{\gamma_{i}} \sum_{i=1}^{n} \frac{1}{\gamma_{i}}+2 \beta^{2} \sigma_{\epsilon}^{6} \sum_{i=1}^{n} \frac{\sigma_{\delta_{i}}^{4}}{\gamma_{i}^{2}} \sum_{i=1}^{n} \frac{1}{\gamma_{i}^{2}} \sum_{i=1}^{n} \frac{1}{\gamma_{i}}+2 k \beta^{2} \sigma_{\epsilon}^{2} \sum_{i=1}^{n} \frac{1}{\gamma_{i}} \sum_{i=1}^{n} \frac{\sigma_{\delta_{i}}^{4}}{\gamma_{i}^{2}} \\
& -2 \beta^{2} \sigma_{\epsilon}^{6}\left[\sum_{i=1}^{n} \frac{\sigma_{\delta_{i}}^{2}}{\gamma_{i}^{2}}\right]^{2} \sum_{i=1}^{n} \frac{1}{\gamma_{i}}-\sigma_{\epsilon}^{6}\left[\sum_{i=1}^{n} \frac{X_{i}}{\gamma_{i}}\right]^{2} \sum_{i=1}^{n} \frac{1}{\gamma_{i}^{2}}-k \sigma_{\epsilon}^{2}\left[\sum_{i=1}^{n} \frac{X_{i}}{\gamma_{i}}\right]^{2}
\end{aligned}
$$

and

$$
\begin{aligned}
E_{2}= & \sigma_{\epsilon}^{4} \sum_{i=1}^{n} \frac{X_{i}^{2}}{\gamma_{i}} \sum_{i=1}^{n} \frac{1}{\gamma_{i}^{2}} \sum_{i=1}^{n} \frac{1}{\gamma_{i}}+k \sum_{i=1}^{n} \frac{X_{i}^{2}}{\gamma_{i}} \sum_{i=1}^{n} \frac{1}{\gamma_{i}}+2 \sigma_{\epsilon}^{4} \beta^{2} \sum_{i=1}^{n} \frac{\sigma_{\delta_{i}}^{4}}{\gamma_{i}^{2}} \sum_{i=1}^{n} \frac{1}{\gamma_{i}^{2}} \sum_{i=1}^{n} \frac{1}{\gamma_{i}} \\
& +2 k \beta^{2} \sum_{i=1}^{n} \frac{\sigma_{\delta_{i}}^{4}}{\gamma_{i}^{2}} \sum_{i=1}^{n} \frac{1}{\gamma_{i}}-2 \beta^{2} \sigma_{\epsilon}^{4}\left[\sum_{i=1}^{n} \frac{\sigma_{\delta_{i}}^{2}}{\gamma_{i}^{2}}\right]^{2} \sum_{i=1}^{n} \frac{1}{\gamma_{i}} \\
& -\sigma_{\epsilon}^{4}\left[\sum_{i=1}^{n} \frac{X_{i}}{\gamma_{i}}\right]^{2} \sum_{i=1}^{n} \frac{1}{\gamma_{i}^{2}}-k\left[\sum_{i=1}^{n} \frac{X_{i}}{\gamma_{i}}\right]^{2} .
\end{aligned}
$$

Note that when $\sigma_{\delta_{i}}^{2}=0, i=1, \cdots, n$, the expression (2.9) is reduced to the variance of the usual model given in (A.5) and when $\sigma_{\delta_{i}}^{2}=\sigma_{\delta}^{2}$ (for all i) the expression (2.9) is also reduced to the variance of the homoscedastic model when $\sigma_{\delta}^{2}$ is known (see eq. (2.12) of ref. [6]).

In order to construct a confidence interval for $X_{0}$ we consider the interval (A.7), where $\hat{V}\left(\hat{X}_{0 C}\right)$ is the estimated variance that follows from (2.9).

\section{Simulation study}

We present a simulation study to compare the performance of the estimators obtained from the heteroscedastic controlled calibration model (Proposed-M) with the results obtained by considering the usual model (Usual-M).

It was considered 3000 samples generated from the Proposed-M. In all the samples, the parameters $\alpha$ and $\beta$ take the values 0.1 and 2 , respectively. The range of values for the controlled variable was $[0,2]$. The fixed values for the controlled variable were $x_{1}=0, x_{i}=x_{i-1}+2 /(n-1), i=2, \cdots, n$, and the parameter values $X_{0}$ were 0.01 (extreme inferior value), 0.8 (near to the central value) and 1.9 (extreme superior value). For the first and second stages we consider the sample of sizes $n=5,20,100,5000$ and $k=2,20,100,500$, respectively. We consider $\sigma_{\epsilon}^{2}=0.04$ and the maximum parameter values of $\sigma_{\delta}^{2}$ as $\max \left\{\sigma_{\delta_{i}}^{2}\right\}_{i=1}^{n}=0.1$. We consider $\sigma_{\delta_{i}}^{2}=i \times 0.1 / n$ for $i=1, \cdots, n$.

The empirical mean bias is given by $\sum_{j=1}^{3000}\left(\hat{X}_{0}-X_{0}\right) / 3000$ and the empirical mean squared error (MSE) is given by $\sum_{j=1}^{3000}\left(\hat{X}_{0}-X_{0}\right)^{2} / 3000$. The 
mean estimated variance of $\hat{X}_{0}$ is given by $\sum_{j=1}^{3000} \hat{V}\left(\hat{X}_{0}\right) / 3000$. The theoretical variances of $\hat{X}_{0}$ is referred to the expressions (A.5) and (2.9) evaluated on the relevant parameter values. In Appendix B it is presented the simulation results.

In Table 1, we observe that, in general, the bias of $\hat{X}_{0}$ from the usual model is smaller than the value supplied by the proposed model, but related to the MSE we have that the outcome from usual model is greater compared with MSE of the proposed model. Also, we observe that the mean estimated variance from the proposed model is closer to the theoretical variance as compared to the outcome from the usual model.

Analyzing Table 2, we observe that the amplitude of the proposed model, in most cases, is smaller when compared with the estimate of the usual model. For all $n$ and $X_{0}$ the amplitude from the usual model greatly decreases as the size of $\mathrm{k}$ increases, this behavior is being reflected on the covering percentage decreasing to less than 95\%. Adopting the correct model we have that when $k$ increases the confidence interval amplitude decreases and the covering percentage increases approaching $95 \%$. 
Table 1: Empirical bias and mean squared error, the mean estimated variance and theoretical variance of $\hat{X}_{0}$.

\begin{tabular}{|c|c|c|c|c|c|c|c|c|c|}
\hline \multirow[t]{2}{*}{$X_{0}$} & \multirow[t]{2}{*}{$\mathrm{n}$} & \multirow[t]{2}{*}{$\mathrm{k}$} & \multicolumn{2}{|c|}{ Usual-M } & \multicolumn{2}{|c|}{ Proposed-M } & \multirow{2}{*}{$\frac{\text { Usual-M }}{\bar{V}\left(\tilde{X}_{0}\right)}$} & \multirow{2}{*}{$\frac{\text { Proposed-M }}{\tilde{V}\left(\tilde{X}_{0}\right)}$} & \multirow{2}{*}{$\begin{array}{c}\text { Theorical variance } \\
\qquad V\left(\dot{X}_{0}\right)\end{array}$} \\
\hline & & & Bias & MSE & Bias & MSE & & & \\
\hline \multirow[t]{13}{*}{0.01} & \multirow[t]{3}{*}{5} & 2 & -0.0156 & 0.0350 & -0.0318 & 0.0334 & 0.0398 & 0.0143 & 0.0257 \\
\hline & & 20 & -0.0236 & 0.0319 & -0.0445 & 0.0278 & 0.0131 & 0.0156 & 0.0211 \\
\hline & & 100 & -0.0183 & 0.0306 & -0.0429 & 0.0276 & 0.0084 & 0.0155 & 0.0207 \\
\hline & \multirow[t]{3}{*}{20} & 2 & -0.0076 & 0.0119 & -0.0049 & 0.0100 & 0.0365 & 0.0053 & 0.0097 \\
\hline & & 20 & -0.0055 & 0.0074 & -0.0073 & 0.0055 & 0.0081 & 0.0036 & 0.0051 \\
\hline & & 100 & -0.0059 & 0.0068 & -0.0101 & 0.0050 & 0.0036 & 0.0033 & 0.0047 \\
\hline & \multirow[t]{3}{*}{100} & 2 & 0.0003 & 0.0063 & 0.0020 & 0.0059 & 0.0315 & 0.0047 & 0.0059 \\
\hline & & 20 & -0.0023 & 0.0019 & -0.0020 & 0.0014 & 0.0046 & 0.0011 & 0.0014 \\
\hline & & 100 & -0.0014 & 0.0015 & -0.0013 & 0.0010 & 0.0017 & 0.0007 & 0.0010 \\
\hline & \multirow[t]{4}{*}{5000} & 2 & 0.0008 & 0.0055 & 0.0008 & 0.0055 & 0.0300 & 0.0050 & 0.0050 \\
\hline & & 20 & 0.0000 & 0.0005 & 0.0000 & 0.0005 & 0.0030 & 0.0005 & 0.0005 \\
\hline & & 100 & -0.0003 & 0.0001 & -0.0004 & 0.0001 & 0.0006 & 0.0001 & 0.0001 \\
\hline & & 500 & 0.0000 & 0.0000 & 0.0000 & 0.0000 & 0.0002 & 0.0000 & 0.0000 \\
\hline \multirow[t]{13}{*}{0.8} & \multirow[t]{3}{*}{5} & 2 & 0.0061 & 0.0193 & 0.0025 & 0.0202 & 0.0254 & 0.0089 & 0.0167 \\
\hline & & 20 & 0.0033 & 0.0135 & -0.0019 & 0.0139 & 0.0047 & 0.0081 & 0.0122 \\
\hline & & 100 & 0.0014 & 0.0132 & -0.0037 & 0.0137 & 0.0029 & 0.0078 & 0.0118 \\
\hline & \multirow[t]{3}{*}{20} & 2 & 0.0015 & 0.0077 & 0.0015 & 0.0077 & 0.0291 & 0.0042 & 0.0074 \\
\hline & & 20 & 0.0016 & 0.0032 & 0.0009 & 0.0032 & 0.0036 & 0.0020 & 0.0029 \\
\hline & & 100 & -0.0005 & 0.0026 & -0.0018 & 0.0026 & 0.0012 & 0.0016 & 0.0025 \\
\hline & \multirow{3}{*}{100} & 2 & 0.0010 & 0.0055 & 0.0014 & 0.0054 & 0.0299 & 0.0044 & 0.0055 \\
\hline & & 20 & 0.0006 & 0.0010 & 0.0007 & 0.0010 & 0.0031 & 0.0008 & 0.0010 \\
\hline & & 100 & -0.0001 & 0.0006 & -0.0001 & 0.0006 & 0.0007 & 0.0004 & 0.0006 \\
\hline & \multirow[t]{4}{*}{5000} & 2 & 0.0014 & 0.0051 & 0.0014 & 0.0051 & 0.0300 & 0.0050 & 0.0050 \\
\hline & & 20 & 0.0006 & 0.0005 & 0.0006 & 0.0005 & 0.0030 & 0.0005 & 0.0005 \\
\hline & & 100 & 0.0001 & 0.0001 & 0.0001 & 0.0001 & 0.0006 & 0.0001 & 0.0001 \\
\hline & & 500 & 0.0000 & 0.0000 & 0.0000 & 0.0000 & 0.0001 & 0.0000 & 0.0000 \\
\hline \multirow[t]{13}{*}{1.9} & \multirow[t]{3}{*}{5} & 2 & 0.0500 & 0.0802 & 0.0582 & 0.0704 & 0.0432 & 0.0275 & 0.0482 \\
\hline & & 20 & 0.0314 & 0.0620 & 0.0503 & 0.0562 & 0.0124 & 0.0278 & 0.0435 \\
\hline & & 100 & 0.0434 & 0.0645 & 0.0594 & 0.0587 & 0.0085 & 0.0283 & 0.0430 \\
\hline & \multirow[t]{3}{*}{20} & 2 & 0.0070 & 0.0213 & 0.0054 & 0.0185 & 0.0351 & 0.0086 & 0.0166 \\
\hline & & 20 & 0.0117 & 0.0160 & 0.0127 & 0.0132 & 0.0075 & 0.0067 & 0.0118 \\
\hline & & 100 & 0.0099 & 0.0161 & 0.0104 & 0.0130 & 0.0033 & 0.0064 & 0.0114 \\
\hline & \multirow[t]{3}{*}{100} & 2 & 0.0016 & 0.0080 & 0.0007 & 0.0076 & 0.0312 & 0.0055 & 0.0074 \\
\hline & & 20 & 0.0019 & 0.0035 & 0.0014 & 0.0029 & 0.0043 & 0.0017 & 0.0028 \\
\hline & & 100 & 0.0001 & 0.0031 & 0.0009 & 0.0025 & 0.0015 & 0.0013 & 0.0024 \\
\hline & \multirow{4}{*}{5000} & 2 & -0.0008 & 0.0051 & -0.0009 & 0.0051 & 0.0300 & 0.0050 & 0.0050 \\
\hline & & 20 & -0.0003 & 0.0006 & -0.0004 & 0.0006 & 0.0030 & 0.0005 & 0.0005 \\
\hline & & 100 & -0.0003 & 0.0002 & -0.0002 & 0.0001 & 0.0006 & 0.0001 & 0.0001 \\
\hline & & 500 & 0.0000 & 0.0001 & 0.0000 & 0.0001 & 0.0001 & 0.0000 & 0.0001 \\
\hline
\end{tabular}

\section{Application}

In this section we illustrate the usefulness of the proposed model by applying it to the data supplied by the chemical laboratory of the "Instituto de Pesquisas Tecnológicas do Estado de São Paulo (IPT)" - Brasil. The outcome from the proposed approach are also compared with the results from the usual model. Our main interest is to estimate the unknown concentration value $X_{0}$ of a sample of the chemical elements such as chromium, cadmium and lead.

Table 3 below presents the fixed values of concentration for the standard solutions with their related uncertainty $\left(u\left(X_{i}\right)\right)$ and the corresponding in- 
Table 2: Covering percentage (\%) and amplitude (A) of the intervals with a $95 \%$ confidence level for the parameter $X_{0}$.

\begin{tabular}{|c|c|c|c|c|c|c|}
\hline \multirow[t]{2}{*}{$X_{0}$} & \multirow[t]{2}{*}{$n$} & \multirow[t]{2}{*}{$k$} & \multicolumn{2}{|c|}{ Usual-M } & \multicolumn{2}{|c|}{ Proposed-M } \\
\hline & & & $\%$ & $A$ & $\%$ & $A$ \\
\hline \multirow[t]{13}{*}{0.01} & \multirow[t]{3}{*}{5} & 2 & 89 & 0.35 & 78 & 0.22 \\
\hline & & 20 & 78 & 0.21 & 89 & 0.24 \\
\hline & & 100 & 70 & 0.17 & 88 & 0.24 \\
\hline & \multirow[t]{3}{*}{20} & 2 & 100 & 0.37 & 74 & 0.13 \\
\hline & & 20 & 95 & 0.17 & 90 & 0.12 \\
\hline & & 100 & 85 & 0.12 & 90 & 0.11 \\
\hline & \multirow[t]{3}{*}{100} & 2 & 100 & 0.35 & 84 & 0.13 \\
\hline & & 20 & 100 & 0.13 & 91 & 0.06 \\
\hline & & 100 & 96 & 0.08 & 90 & 0.05 \\
\hline & \multirow[t]{4}{*}{5000} & 2 & 100 & 0.34 & 94 & 0.14 \\
\hline & & 20 & 100 & 0.11 & 95 & 0.04 \\
\hline & & 100 & 100 & 0.05 & 94 & 0.02 \\
\hline & & 500 & 100 & 0.02 & 94 & 0.01 \\
\hline \multirow[t]{13}{*}{0.8} & \multirow[t]{3}{*}{5} & $\overline{2}$ & 90 & 0.28 & 78 & 0.18 \\
\hline & & 20 & 73 & 0.13 & 87 & 0.17 \\
\hline & & 100 & 63 & 0.10 & 87 & 0.17 \\
\hline & \multirow[t]{3}{*}{20} & 2 & 100 & 0.33 & 73 & 0.11 \\
\hline & & 20 & 95 & 0.12 & 87 & 0.09 \\
\hline & & 100 & 81 & 0.07 & 88 & 0.08 \\
\hline & \multirow[t]{3}{*}{100} & 2 & 100 & 0.34 & 86 & 0.12 \\
\hline & & 20 & 100 & 0.11 & 91 & 0.05 \\
\hline & & 100 & 97 & 0.05 & 89 & 0.04 \\
\hline & \multirow[t]{4}{*}{5000} & 2 & 100 & 0.34 & 95 & 0.14 \\
\hline & & 20 & 100 & 0.11 & 95 & 0.04 \\
\hline & & 100 & 100 & 0.05 & 95 & 0.02 \\
\hline & & 500 & 100 & 0.02 & 93 & 0.01 \\
\hline \multirow[t]{13}{*}{1.9} & \multirow[t]{3}{*}{5} & $\overline{2}$ & 78 & 0.35 & 81 & 0.31 \\
\hline & & 20 & 59 & 0.20 & 86 & 0.32 \\
\hline & & 100 & 51 & 0.17 & 87 & 0.32 \\
\hline & \multirow[t]{3}{*}{20} & 2 & 98 & 0.36 & 78 & 0.17 \\
\hline & & 20 & 81 & 0.17 & 84 & 0.16 \\
\hline & & 100 & 62 & 0.11 & 84 & 0.16 \\
\hline & \multirow[t]{3}{*}{100} & 2 & 100 & 0.34 & 87 & 0.14 \\
\hline & & 20 & 97 & 0.13 & 87 & 0.08 \\
\hline & & 100 & 83 & 0.08 & 84 & 0.07 \\
\hline & \multirow[t]{4}{*}{5000} & 2 & 100 & 0.34 & 95 & 0.14 \\
\hline & & 20 & 100 & 0.11 & 94 & 0.04 \\
\hline & & 100 & 100 & 0.05 & 93 & 0.02 \\
\hline & & 500 & 99 & 0.02 & 89 & 0.01 \\
\hline
\end{tabular}

tensities for the chromium, cadmium and lead elements. The uncertainties considered are computed using the method recommended by the ISOGUM guide (see [11]) and the intensities are supplied by the plasma spectrometry method. This data is referred to the first stage of the heteroscedastic controlled calibration model.

Moreover, Table 4 below presents the intensities of the sample solutions of chromium, cadmium and lead elements. These data are referred to as the second stage of the calibration model.

Observing Tables 3 and 4 we verify that the uncertainty values increase with the concentration values.

We consider $\sigma_{\delta_{i}}^{2}=u\left(X_{i}\right)^{2}$. The expanded uncertainty $U\left(X_{0}\right)$ is obtained 
Table 3: Concentration $(m g / g)$, uncertainty $\left(u\left(X_{i}\right)\right)$ and intensity of the standard solutions of chromium, cadmium and lead elements.

\begin{tabular}{|c|r|r|r|r|r|r|r|r|}
\hline \multicolumn{3}{|c|}{ Chromium element } & \multicolumn{3}{c|}{ Cadmium element } & \multicolumn{3}{|c|}{ lead element } \\
\hline$X_{i}$ & $u\left(X_{i}\right)$ & Intensity & $X_{i}$ & $u\left(X_{i}\right)$ & Intensity & $X_{i}$ & $u\left(X_{i}\right)$ & Intensity \\
\hline 0.05 & 0.00016 & 6455.900 & 0.05 & 0.00016 & 4.89733 & 0.05 & 0.00015 & 0.9471 \\
0.11 & 0.00027 & 13042.933 & 0.10 & 0.00027 & 9.706 & 0.10 & 0.00025 & 1.46833 \\
0.26 & 0.00040 & 32621.733 & 0.25 & 0.00041 & 23.41333 & 0.26 & 0.00039 & 3.09033 \\
0.79 & 0.00122 & 97364.500 & 0.73 & 0.00122 & 69.73 & 0.77 & 0.00117 & 8.40533 \\
1.05 & 0.00161 & 129178.100 & 1.01 & 0.00168 & 96.85667 & 1.01 & 0.00155 & 10.92667 \\
\hline
\end{tabular}

Table 4: Intensity of the sample solutions of chromium, cadmium and lead elements.

\begin{tabular}{|c|c|c|}
\hline Chromium element & Cadmium element & Lead element \\
\hline 10173.6 & 5.066 & 1.303 \\
10516.9 & 5.027 & 1.290 \\
10352.2 & 5.085 & 1.341 \\
\hline
\end{tabular}

multiplying the squared root of the estimate of variance of $\hat{X}_{0}$ by the value 1.96 (see [2] and [8]).

We use the optim command from R-project program to estimate the parameters $\beta$ and $\sigma_{\epsilon}^{2}$ on the likelihood function of the proposed model (2.6). We use as initial point the estimates from $\hat{\beta}=\sum_{i=1}^{n}\left(X_{i}-\bar{x}\right)\left(Y_{i}-\bar{Y}\right) / \sum_{i=1}^{n}\left(X_{i}-\right.$ $\bar{X})^{2}$ and $\hat{\sigma}_{\epsilon}^{2}=\sum_{i=1}^{n}\left(Y_{0 i}-\bar{Y}_{0}\right)^{2} / n$, which are the estimators from the homoscedastic controlled calibration model when $\sigma_{\delta}^{2}$ is unknow [6].

Table 5 presents estimates of $\alpha, \beta, X_{0}, V\left(\hat{X}_{0}\right)$ and the expanded uncertainty, $U\left(X_{0}\right)$, from the proposed model (Proposed-M) of chromium, cadmium and lead elements. Also, we present the estimates obtained from usual calibration model (Usual-M) to observe the performance of both models.

In Table 5, for cadmium and lead elements, we observe that the estimates of $\alpha, \beta$ and $X_{0}$ from the Proposed-M and Usual-M are the same. For the chromium element, there are small differences. Also, we observe that for the chromium element there is a small difference between the estimates of $X_{0}$ and $U\left(X_{0}\right)$ respectively obtained from the usual model and the proposed model. Despite the relevant estimates of $\alpha, \beta$ and $X_{0}$ from both approaches for cadmium and lead element match, the estimates of $V\left(X_{0}\right)$ and $U\left(X_{0}\right)$ differ considerably, the estimates obtained adopting the usual model is greater than the estimates outcome supplied by the proposed model.

\section{Concluding remarks}

The expanded uncertainty of $X_{0}$ from the proposed model arises from the errors appearing in the both process, the reading of equipment and the heteroscedastic error in the preparation of standard solutions. We observe that, 
Table 5: Estimates of $\alpha, \beta, X_{0}, V\left(\hat{X}_{0}\right)$ and $U\left(X_{0}\right)$ related to usual and heteroscedastic model, for the chromium, cadmium and lead element.

\begin{tabular}{|c|c|c|c|c|c|c|}
\hline \multirow{2}{*}{ Parameters } & \multicolumn{2}{|c|}{ Chromium element } & \multicolumn{2}{c|}{ Cadmium element } & \multicolumn{2}{c|}{ Lead element } \\
\cline { 2 - 7 } & Usual-M & Proposed-M & Usual-M & Proposed-M & Usual-M & Proposed-M \\
\hline$\alpha$ & 134.9469 & 124.2801 & 0.454801 & 0.454801 & -0.3822126 & -0.3822126 \\
$\beta$ & 123003.7 & 123027.3 & 10.54381 & 10.54381 & 94.29881 & 94.29881 \\
$X_{0}$ & 0.08302691 & 0.08309769 & 0.08123556 & 0.08123556 & 0.05770535 & 0.05770535 \\
$V\left(\hat{X}_{0}\right)$ & $4.357870 \mathrm{e}-06$ & $4.474395 \mathrm{e}-06$ & $7.898643 \mathrm{e}-05$ & $4.440342 \mathrm{e}-06$ & 0.0001181068 & $7.237226 \mathrm{e}-08$ \\
$U\left(X_{0}\right)$ & 0.004091601 & 0.004145942 & 0.01741936 & 0.004130135 & 0.02130068 & 0.000527281 \\
\hline
\end{tabular}

despite the classical model only considers the error originated from equipment reading, there are some applications in which the expanded uncertainty is greater than the one obtained through the new approach.

Various aspects of the model studied above deserve attention in future research, e.g. it is not considerated the error arisen from the test sample solution preparation, the proposed model can be studied by considering other type of distribution of the errors, such as skew normal distribution [12]. In particular, one of the drawbacks of the usual model is that it does not consider the error in the independent variable, we believe that despite that this error being very small, it must be considered as an important property of the calibration model. We will concentrate on one of the problems described above in a future work.

\section{Acknowledgments}

The authors are grateful to Prof. Dr. Heleno Bolfarine for carefully reading the manuscript and Dr. Olga Satomi from "Instituto de Pesquisas Tecnológicas" - IPT. Betsabé G. B. Achic has been supported by a grant from CNPq. 


\section{A Usual calibration model}

The first and second stage equations of the usual linear calibration model are given, respectively, by

$$
\begin{aligned}
Y_{i} & =\alpha+\beta x_{i}+\epsilon_{i}, \quad i=1,2 \cdots, n, \\
Y_{0 i} & =\alpha+\beta X_{0}+\epsilon_{i}, \quad i=n+1, n+2, \cdots, n+k .
\end{aligned}
$$

It is considered the following assumptions:

- $x_{1}, x_{2}, \cdots, x_{n}$ take fixed values, which are considered as true values.

- $\epsilon_{1}, \epsilon_{2}, \cdots, \epsilon_{n+k}$ are independent and normally distributed with mean 0 and variance $\sigma_{\epsilon}^{2}$.

The model parameters are $\alpha, \beta, X_{0}$ and $\sigma_{\epsilon}^{2}$ and the main interest is to estimate the quantity $X_{0}$.

The maximum likelihood estimators of the usual calibration model are given by

$$
\begin{aligned}
\hat{\alpha} & =\bar{Y}-\hat{\beta} \bar{x}, \quad \hat{\beta}=\frac{S_{x Y}}{S_{x x}}, \quad \hat{X}_{0}=\frac{\bar{Y}_{0}-\hat{\alpha}}{\hat{\beta}}, \\
\sigma_{\epsilon}^{2} & =\frac{1}{n+k}\left[\sum_{i=1}^{n}\left(Y_{i}-\hat{\alpha}-\hat{\beta} x_{i}\right)^{2}+\sum_{i=n+1}^{n+k}\left(Y_{0 i}-\bar{Y}_{0}\right)^{2}\right],
\end{aligned}
$$

where

$$
\begin{aligned}
\bar{x} & =\frac{1}{n} \sum_{i=1}^{n} x_{i}, \quad \bar{Y}=\frac{1}{n} \sum_{i=1}^{n} Y_{i}, \quad S_{x Y}=\frac{1}{n} \sum_{i=1}^{n}\left(x_{i}-\bar{x}\right)\left(Y_{i}-\bar{Y}\right), \\
S_{x x} & =\frac{1}{n} \sum_{i=1}^{n}\left(x_{i}-\bar{x}\right)^{2}, \quad \bar{Y}_{0}=\frac{1}{n} \sum_{i=n+1}^{n+k} Y_{0 i} .
\end{aligned}
$$

The approximation of order $n^{-1}$ for the variance of $\hat{X}_{0}$ is given by

$$
V_{1}\left(\hat{X}_{0}\right)=\frac{\sigma_{\epsilon}^{2}}{\beta^{2}}\left[\frac{1}{k}+\frac{1}{n}+\frac{\left(\bar{x}-X_{0}\right)^{2}}{n S_{x x}}\right] .
$$

In order to construct a confidence interval for $X_{0}$, we consider that

$$
\frac{\hat{X}_{0}-X_{0}}{\sqrt{\hat{V}\left(\hat{X}_{0}\right)}} \stackrel{D}{\longrightarrow} N(0,1)
$$

hence, the approximated confidence interval for $X_{0}$ with a confidence level $(1-\alpha)$, is given by

$$
\left(\hat{X}_{0}-z_{\frac{\alpha}{2}} \sqrt{\hat{V}\left(\hat{X}_{0}\right)}, \hat{X}_{0}+z_{\frac{\alpha}{2}} \sqrt{\hat{V}\left(\hat{X}_{0}\right)}\right),
$$

where $z_{\frac{\alpha}{2}}$ is the quantile of order $\left(1-\frac{\alpha}{2}\right)$ of the standard normal distribution. 


\section{References}

[1] Shukla, G.K. On the problem of calibration. Technometrics 14 (1972) 547.

[2] EURACHEM/CITAC Guide(2000) Quantifying uncertainty in analytical measurement, 2nd edn., Final Draft April 2000. EURACHEM: http://www.measurementuncertainty.org

[3] S. H. Chui, Q; Zucchini R. R; and Lichtig J. Qualidade de medições em química analítica. Estudo de caso: determinação de cádmio por espectrometria de absorção atômica com chama.Química Nova 24 (2001) 374 .

[4] Bruggemann, L. and Wenrich R. Evaluation of measurement uncertainty for analytical procedures using a linear calibration function. Accreditation and Quality Assurance 7 (2002) 269.

[5] Berkson, J. Are there two regression?. Journal of the American Statistical Association 45 (1950) 164.

[6] Blas, B. G; Sandoval, M. C; Satomi, O. Homoscedastic controlled calibration model. Journal of Chemometrics 21 (2007) 145.

[7] Ballico, M. Limitations of the Welch-Satterthwaite approximation for measurement uncertainty calculations. Metrologia 37 (2000) 61.

[8] Blas, B. G.(2005). Calibração controlada aplicada à química analítica. São Paulo: IME-USP. Dissertação de Mestrado.

[9] Tallis G.M. Note on a Calibration problem.Biometrika 56 (1969) 505.

[10] Lwin, T. and Maritz J. S. A note on the problem of statistical calibration. Applied Statistics 29 (1980) 135.

[11] International Organization for Standardization (1995). Guide to the expression of uncertainty in measurement (ISOGUM) .

[12] Azzalini, A. A class of distributions which includes the normal ones. Scandinavian Journal of Statistics 12 (1985) 171. 